\title{
Regulierung von Arzneimitteln für neuartige Therapien durch den Gemeinsamen Bundesausschuss
}

\section{JOSEF HECKEN}

Prof. Josef Hecken ist unparteiischer Vorsitzender des Gemeinsamen Bundesausschusses (G-BA), Berlin

\author{
Mit den sogenannten „Advanced Therapy \\ Medicinal Products (ATMPs)" bricht eine neue \\ Ära in der Behandlung von Krankheiten an, die \\ gleichzeitig mit extrem hohen Kosten für das \\ deutsche Gesundheitssystem verbunden ist. Im \\ internationalen Kontext wird schon länger über \\ alternative Erstattungsmodelle für diese Therapien \\ diskutiert, um ein angemessenes Verhältnis \\ zwischen Kosten und Nutzen der Therapie zu \\ schaffen. Mit dem Gesetz für mehr Sicherheit in der \\ Arzneimittelversorgung sind die Grundlagen gelegt, \\ um dem G-BA die notwendige Regulierung der ATMPs \\ unter den Gesichtspunkten der Patientensicherheit \\ und qualitätsgesicherten Versorgung zu ermöglichen.
}

\section{Einleitung}

Mit den sogenannten „Advanced Therapy Medicinal Products (ATMPs)“ bricht eine neue Ära in der Behandlung von Krankheiten an, die gleichzeitig mit extrem steigenden Kosten für das deutsche Gesundheitssystem verbunden ist. $\mathrm{Zu}$ den Arzneimitteln für neuartige Therapien gehören die drei Gruppen Gentherapeutika, somatische Zelltherapien und biotechnologisch bearbeitete Gewebeprodukte. Insgesamt sind derzeit neun ATMPs zentral in der EU zugelassen (Stand: Februar 2019): fünf Gentherapien, zwei somatische Zelltherapien sowie zwei biotechnologisch bearbeitete Gewebeprodukte.

Unter den fünf Gentherapien befinden sich seit August 2018 die zwei CAR-TZelltherapien Kymriah® und Yescarta®. Die CAR-T-Zell-Therapie (Chimeric Antigen Receptor T-Zell-Therapie) ist eine neue Form der Immuntherapie bei Krebspatienten, die auf gentechnische Veränderung der Immunzellen der Patientinnen und Patienten beruht. Die Genmodifizierten T-Zellen können Antigene auf der Oberfläche von malignen Zellen erkennen und eine Immunantwort zur Abtötung der malignen Zellen initiieren. Ebenso wie schon bei der Einführung der Biologika werden Gentherapien die Behandlung in vielen Bereichen revolutionieren. Insbesondere, weil dadurch oftmals mit einer einmaligen Anwendung beachtliche Behandlungserfolge erreicht werden können.

Aufgrund der sehr hohen Kosten zu einem bestimmten Zeitpunkt sind die bereits heute zur Verfügung stehenden Gentherapien mit großen finanziellen Herausforderungen für das Gesundheitssystem verbunden. Für die Gentherapie Kymriah® zur Behandlung bei Leukämie und Lymphomen betragen die Kosten für die einmalige 
Verabreichung zurzeit 320.000 Euro. Für das Konkurrenzprodukt Yescarta ${ }^{\circledR}$, das zeitgleich mit Kymriah® zur Behandlung von Lymphomen zugelassen wurde, liegen die Kosten bei etwa 390.000 Euro. Dies umfasst ausschließlich die Kosten für das Arzneimittel, unberücksichtigt bleiben dabei unter anderem die notwendigen Kosten für die stationäre Unterbringung der Patientinnen und Patienten sowie das Nebenwirkungsmanagement, das aufgrund der häufig auftretenden, teils

\section{Der komplexe Herstellungsprozess von Gentherapeutika und kleine Patientenzahlen erschweren die Umsetzung randomisierter klinischer Studien.}

schwerwiegenden Nebenwirkungen kurz nach der Verabreichung der CAR-T-Zellen notwendig ist. Die Gentherapie Luxturna® zur Behandlung des Sehverlustes liegt mit Kosten von voraussichtlich 410.550 Euro pro Auge nochmals höher. Die erreichten Preisdimensionen pro Patientin oder Patient übertreffen damit um ein Vielfaches die Kosten des in der gesetzlichen Krankenversicherung (GKV) umsatzstärksten Arzneimittels Humira ${ }^{\circledR}$, für das Jahrestherapiekosten zwischen 20.000 Euro und 40.000 Euro anfallen. Dabei sind zum Zeitpunkt der Markteinführung zumeist der langfristige Nutzen und das mögliche Heilungspotenzial dieser neuen Therapien unklar. So zeigen Studiendaten zu den CAR-T-Zellen Kymriah® und Yescarta ${ }^{\circledR}$, dass eine erneute Gabe der CAR-T-Zellen unter bestimmten Voraussetzungen bei Versagen der Therapie nicht ausgeschlossen ist und weitere Kosten für das Gesundheitssystem verursachen kann.

Die Anzahl klinischer Studien mit ATMPs, insbesondere Gentherapien, steigt stetig an. Zurzeit befinden sich bereits weitere mit sehr hohen Kosten verbundene Gentherapien, beispielsweise der Wirkstoff LentiGlobin zur Behandlung der ß-Thalassämie und der Wirkstoff AVXS-101 zur Behandlung der spinalen Muskelatrophie, im Zulassungsverfahren bei der europäischen Zulassungsbehörde. Für AVXS-101 hat der zuständige pharmazeutische Unternehmer Novartis/AveXis angekündigt, dass ein Preis von 4 bis 5 Millionen US-Dollar pro Patientin oder Patient kosteneffektiv sei.

Im Hinblick auf die demografische Entwicklung mit der zunehmenden Lebenserwartung der Bevölkerung ist auch mit einem Anstieg der chronischen, meist im Alter auftretenden Krankheiten wie Arthrose, Alzheimer oder Morbus Parkinson, aber auch onkologischen Erkrankungen sowie Augen- und Herzerkrankungen zu rechnen. Das deutsche Gesundheitssystem muss sich also auf extrem stark steigende Kosten vorbereiten, sollten in Zukunft immer mehr ähnlich innovative Therapien zur Verfügung stehen.

\section{Qualitätsgesicherte Anwendung und Generierung von Evidenz}

Momentan zielen Gentherapien noch häufig auf speziell selektierte Patientenpopulationen ab, die einen bestimmten Krankheitsmarker wie beispielsweise eine spezifische Genmutation aufweisen. Dies führt dazu, dass ATMPs derzeit überwiegend für seltene Leiden entwickelt werden. Von einer seltenen Krankheit spricht man, wenn nur 5 oder weniger von 10.000 Personen in einem Land der EU davon betroffen sind. Der komplexe Herstellungsprozess und die kleinen Patientenzahlen haben wiederum zur Folge, dass randomisierte klinische Studien häufig schwieriger umzusetzen sind als bei konventionellen Arzneimitteln. So lag nur bei 3 der 9 zugelassenen ATMPs eine randomisierte kontrollierte Studie der Zulassung zugrunde. Die vorhandene Evidenz zu AMTPs bei Marktzugang ist deshalb oftmals nicht sehr belastbar, und es ist unklar, ob wirksame und vor allem sichere Therapien vorliegen.

Der Gesetzgeber hat mit dem Gesetz für mehr Sicherheit in der Arzneimittelversorgung (GSAV) bereits erste Konsequenzen gezogen, indem er den Gemeinsamen Bundesausschuss (G-BA) nach dem Gesetzentwurf dazu ermächtigt, bei schwacher Evidenzlage zum Zeitpunkt der Nutzenbewertung anwendungsbegleitende weitere Evidenzgenerierung zu fordern, um auf der Basis der sich dadurch verbessernden „bestverfügbaren Evidenz“ Nachbewertungen vornehmen zu können. Ebenso kann nach dem Entwurf des GSAV die Anwendung von Arzneimitteln für neuar-

\section{Abbildung 1: Anforderungen an qualifizierte Behandlungseinrichtungen}

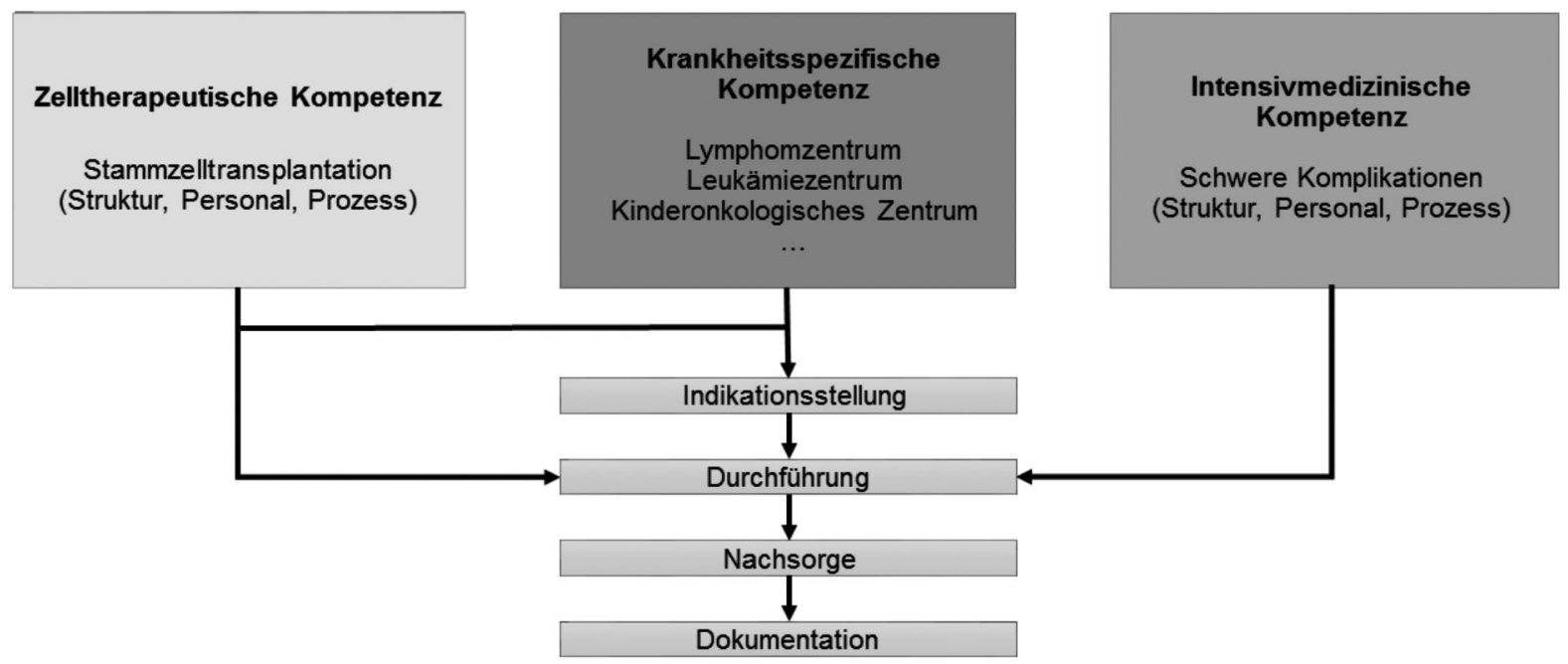

Quelle: Eigene Darstellung 
tige Therapien an strenge Limitationen zur Gewährleistung des Qualitätsgebotes und des Patientenschutzes gebunden werden.

Ein erstes Beispiel für die mögliche Ausgestaltung einer dementsprechenden Richtlinie lieferte der G-BABeschluss zu dem CAR-TZellprodukt Kymriah® vom 7. März 2019. Darin wurden im Rahmen der qualitätsgesicherten Anwendung unter Berücksichtigung der übereinstimmenden Empfehlungen der sachverständigen Organisationen und Personen der medizinischen Wissenschaft und Praxis Anforderungen an die Qualifikation der Behandlungseinrichtung sowie die Infrastruktur und Organisation definiert.

Diese beinhalten zum einen die notwendige umfangreiche Erfahrung der Behandlungseinrichtungen in der Behandlung der jeweiligen malignen Grunderkrankung der Leukämie bzw. Lymphome, die in Form von Mindestfallzahlen an Behandlungsfällen darzulegen ist, und zum anderen die umfangreiche Erfahrung in der Zelltherapie, die durch die Meldung einer bestimmten Anzahl an durchgeführten Stammzelltransplantationen an das Deutsche Register für Stammzelltransplantationen (DRST) oder das European Bone Marrow Transplantation Registry (EBM-
TR) dokumentiert wird. Darüber hinaus wurden unter anderem weitere personelle und fachliche Anforderungen an den behandelnden Arzt bzw. die behandelnde Ärztin und das zuständige Pflegepersonal,

für Stammzelltransplantationen, im Pädiatrischen Register für Stammzelltransplantationen (PRST) oder im Register der European Society for Blood and Marrow Transplantation (EBMT) und für die zeitnahe Dokumentation vorhalten müssen. Die Beschränkung der Anwendung auf qualifizierte Behandlungseinrichtungen, welche eine adäquate Anbindung an Registermodule und eine hohe Compliance in der Meldung entsprechender Daten aufweisen, kann wesentlich dazu beitragen, die Relevanz von Registerdaten sowohl für die Nutzenbewertung als auch für alternative Erstattungsmodelle zu steigern.

die notwendigen infrastrukturellen bzw. organisatorischen Voraussetzungen für eine Behandlung mit Kymriah® sowie der intensivmedizinischen Versorgung der Patienten festgelegt.

Die Beschränkung der Anwendung des Arzneimittels auf definierte qualifizierte Behandlungseinrichtungen soll zudem dabei helfen, aussagekräftigere weitergehende Evidenz nach der Zulassung zu generieren. So wurde im Rahmen der qualitätsgesicherten Anforderungen zu Kymriah® definiert, dass die Einrichtungen personelle und strukturelle Voraussetzungen für den Anschluss an die geplanten Registermodule für CAR-T-Zellen im Deutschen Register

\section{Erstattungsmodelle für ATMPs}

Die einmalig verabreichten, sehr kostenintensiven Gentherapien stellen eine große Herausforderung für das deutsche Gesundheitssystem dar. Unter Berücksichtigung des unklaren langfristigen Nutzens dieser Therapien zum Zeitpunkt des Marktzugangs beinhalten diese einmalig anfallenden hohen Kosten ein großes Risiko für die Kostenträger, insbesondere für kleinere Krankenkassen. Im internationalen Kontext wird schon länger über alternative Erstattungsmodelle für diese Therapien diskutiert, um ein angemessenes Verhältnis zwischen Kosten und Nutzen der Therapie zu schaffen.

\section{Abbildung 2: Schematische Darstellung der Einmalzahlung für kostenintensive Gentherapien mit unklarem langfristigen Nutzen}
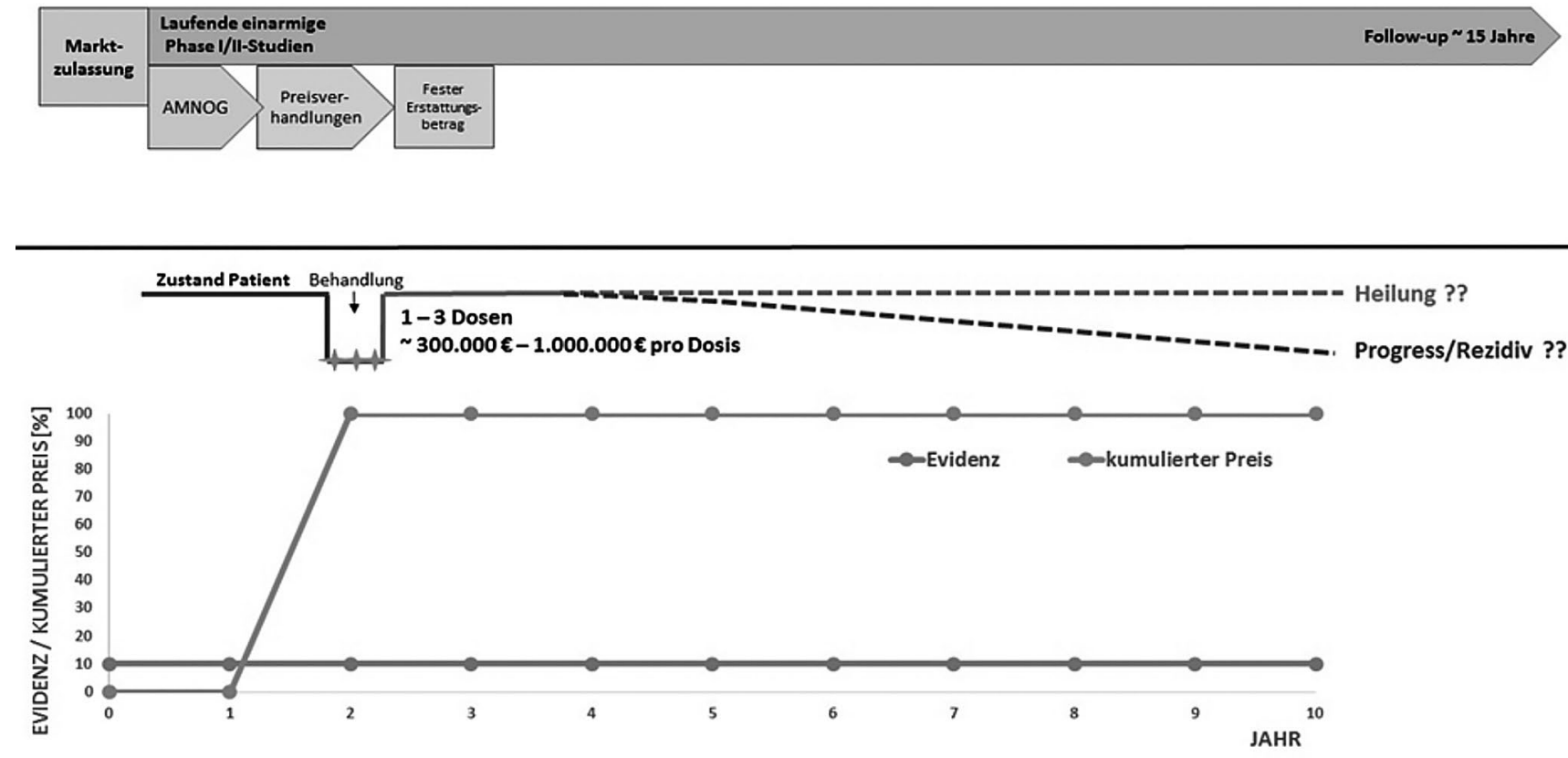

Quelle: Eigene Darstellung 
Ein häufig diskutiertes Modell ist das Pay-for-Performance-Schema (P4PSchema). Dabei werden konkrete Behandlungsziele bzw. Behandlungserfolge definiert, die zu einem bestimmten Zeitpunkt oder nach einem bestimmten Zeitraum erreicht werden müssen. Tritt der Behandlungserfolg nicht ein, zahlt der pharmazeutische Unternehmer einen Teil des Erstattungsbetrages oder den gesamten Erstattungsbetrag an die Kostenträger zurück.

Ein alternatives Modell stellt die Annuitätenzahlung dar, bei der der Preis der Therapie über einen definierten Zeitraum aufgeteilt wird. Die Fortzahlung der Therapie kann hierbei an den Behandlungserfolg der Patientin oder des Patienten gekoppelt werden. Solange die Behandlung wirksam ist, wird die Annuitätenzahlung fortgesetzt, sobald die Patientin oder der Patient einen Rückfall bzw. ein Fortschreiten der Erkrankung zeigt, stoppt die Fortzahlung.

Die größte Schwierigkeit bei einer an den Behandlungserfolg gekoppelten Erstattung ist die Frage, wie genau der Behandlungserfolg für die jeweilige Indikation und Krankheitssituation (z.B.
Schweregrad der Erkrankung) objektiv definiert werden kann. So ist nicht klar, ob für jede Indikation objektive Parameter existieren, die zur Messung des Behandlungserfolges eingesetzt werden können. Darüber hinaus müssten diese Parameter im Rahmen von Routinedaten für die Kostenträger erfassbar sein.

Während in Deutschland bislang nur wenige solcher alternativen Erstattungsmodelle vorhanden sind, existieren international, insbesondere in den USA, einige Beispiele dafür. Für die CAR-T-Therapie Kymriah ${ }^{\circledR}$ basierte das P4PSchema in den USA auf dem Ansprechen der Patientin oder des Patienten auf die Therapie innerhalb eines Monats nach Infusion der CAR-T-Zellen. In Deutschland hat der pharmazeutische Unternehmer

Novartis mit mittelgroßen Kassen eine Kostenrückzahlung vereinbart, sofern der mit Kymriah® behandelte Patient bzw. die Patientin nicht überlebt.

Ein Annuitätenmodell wurde Anfang Januar 2018 von dem Entwickler der Gentherapie Luxturna ${ }^{\circledR}$, Spark Thera- peutics, in den USA abgeschlossen. Hierbei wird die Zahlung für Luxturna ${ }^{\circledR}$ auf einen längeren Zeitraum ausgedehnt, wobei die Kostenträger bei einem fehlenden Eintreten des Behandlungserfolgs eine Rückerstattung erhalten.

Im GSAV ist die Idee angelegt, weitergehende Evidenz nach Marktzulassung zu generieren und davon abhängig den Erstattungsbetrag anzupassen. Die Verknüpfung zwischen Erstattung und

\section{Die Erfahrung in der Nutzenbewertung von ATMPs wird aufgrund der großen Dynamik dieses Therapiesektors zukünftig stark wachsen.}

Generierung weiterer Evidenz kann dazu beitragen, Evidenzlücken zu schließen und gegebenenfalls konkrete Behandlungserfolge für die Umsetzung erfolgsabhängiger Erstattungsmodelle besser zu definieren.

Der G-BA wird ermächtigt, in bestimmten Fällen bei noch ausstehenden

\section{Abbildung 3: Schematische Darstellung eines möglichen Annuitätenmodells für die Erstattung kostenintensiver Gentherapien mit Verknüpfung an den Behandlungserfolg der Patientin/des Patienten}
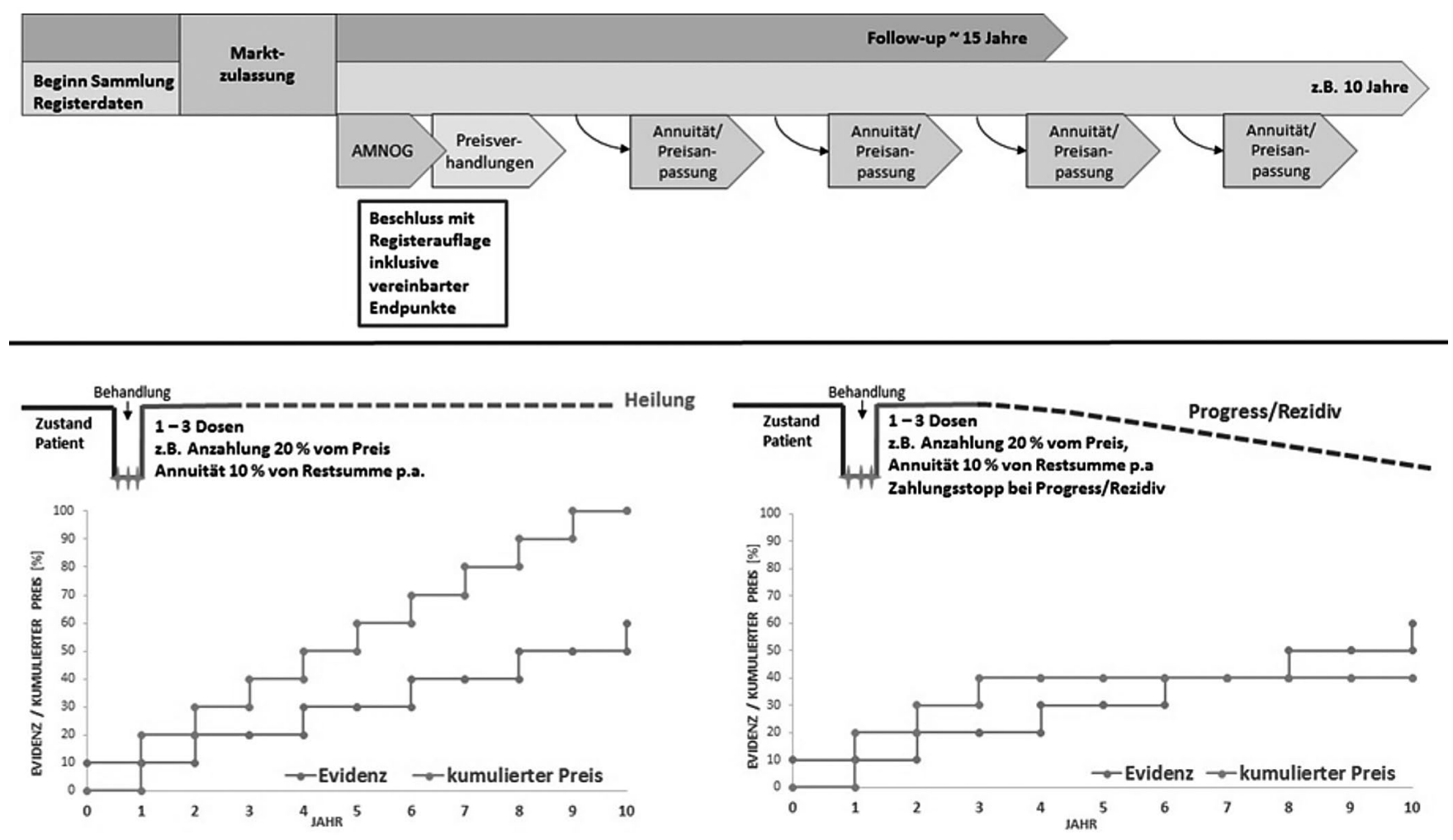

Quelle: Eigene Darstellung 
Daten zum Zeitpunkt der Nutzenbewertung (z.B. bei Orphan Drugs, bedingten Zulassungen) eine anwendungsbegleitende Datenerhebung vom pharmazeutischen Unternehmer innerhalb einer angemessenen Frist zum Zweck der Nutzenbewertung zu fordern.

Die Befugnis zur Verordnung des Arzneimittels zulasten der GKV kann zudem auf solche Vertragsärztinnen und Vertragsärzte oder zugelassene Krankenhäuser beschränkt werden, die an der anwendungsbegleitenden Datenerhebung mitwirken.

Die Verpflichtung zur Teilnahme an der Datenerhebung ist erforderlich, um bei der häufig geringen Anzahl an Patientinnen und Patienten, die mit diesen Arzneimitteln versorgt werden, eine umfassende Datengrundlage für eine erneute Bewertung dieser Arzneimittel zu erhalten.

Eine generelle Beschränkung der Verordnungsfähigkeit dieser Arzneimittel auf Einrichtungen bzw. Zentren ist damit nicht verbunden.
Der Erstattungsbetrag ist bei Arzneimitteln mit bedingter Zulassung oder einer Zulassung unter außergewöhnlichen Umständen sowie bei Arzneimitteln zur Behandlung eines seltenen Leidens regelmäßig neu zu verhandeln, sobald eine vom G-BA gesetzte Frist für die Durchführung einer Datenerhebung verstrichen ist und der G-BA einen erneuten Beschluss zur Nutzenbewertung gefasst hat.

Die anwendungsbegleitende Datenerhebung könnte auf Basis von Registern aufgebaut werden. Um die Generierung weitergehender aussagekräftiger Evidenz nach Markteinführung zu fördern, hat der G-BA in der qualitätsgesicherten Anwendung von Kymriah® entsprechend definiert, dass die qualifizierte Behandlungseinrichtung personelle und strukturelle Voraussetzungen für den Anschluss an die geplanten Registermodule für CAR-T-Zellen im Deutschen Register für Stammzelltransplantationen (DRST), im Pädiatrischen Register für Stammzelltransplantationen (PRST) oder im Register der European Society for Blood and Marrow Transplantation (EBMT) und für die zeitnahe Dokumentation vorhalten muss.

\section{Fazit}

Die Erfahrung in der Nutzenbewertung von ATMPs wird aufgrund der großen Dynamik dieses Therapiesektors zukünftig stark wachsen. Mit dem GSAV sind viele Ansatzpunkte angelegt, die eine sinnvolle Regulierung der ATMPs unter dem Gesichtspunkt der Patientensicherheit und qualitätsgesicherten Versorgung ermöglichen. Zudem werden mit Inkrafttreten des GSAV und der dort verankerten möglichen anwendungsbegleitenden Datenerhebung und Neu-Verhandlung des Erstattungspreises in Abhängigkeit der erhobenen Daten weitere Möglichkeiten für erfolgsabhängige Erstattungsmodelle geschaffen werden.

\title{
Quantifizierung des volkswirtschaftlichen Beitrags der Gesundheitswirtschaft in den Bundesländern Deutschlands
}

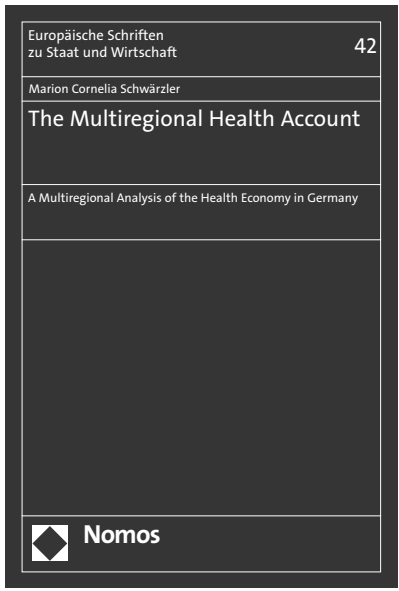

\author{
The Multiregional Health Account \\ A Multiregional Analysis of the Health Economy in Germany \\ Von Dr. Marion Cornelia Schwärzler \\ 2019, 227 S., brosch., 49,-€ \\ ISBN 978-3-8487-5655-1 \\ (Europäische Schriften zu Staat und Wirtschaft, Bd. 42) \\ nomos-shop.de/40909
}

Im Fokus dieses Bandes steht die Gesundheitswirtschaftliche Gesamtrechnung für die Bundesländer Deutschlands. Neben den methodischen Aspekten zur Quantifizierung des ökonomischen Beitrags der Gesundheitswirtschaft werden die Ergebnisse in den Kontext der Krankenhausinvestitionen gesetzt.

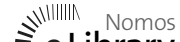

今 e Library Unser Wissenschaftsprogramm ist auch online verfügbar unter: www.nomos-elibrary.de
} 